\title{
The Causation of "Bisphosphonate-Associated" Atypical Femur Fractures
}

\author{
William Banks Hinshaw* \\ Department of Surgery, Harris Regional Hospital, Sylva, North Carolina \\ *Corresponding author: William Banks Hinshaw, Department of Surgery, Harris Regional Hospital, North Carolina
}

"Some...heretofore moved by the reasons of Galen and Colombo...will yeeld."

William Harvey 1628

ARTICLE INFO
Received: 慧 February 17, 2020
Published: 彗 February 25, 2020

Citation: William Banks Hinshaw. The Causation of "Bisphosphonate-Associated" Atypical Femur Fractures. Biomed J Sci \& Tech Res 25(5)-2020. BJSTR. MS.ID.004276.

Keywords: Bisphosphonate; Atypical Fracture; Femur; Osteonecrosis; Causation

\begin{abstract}
The last decades of the $20^{\text {th }}$ century saw the development of intense research programs directed toward the reduction of low-trauma fracture risk by the use of preventative medications. It is not the intention here to recite the history of these efforts, as these have been amply described elsewhere. After the turn of the century, it became apparent that the introduction of the bisphosphonate medications for the purpose was associated with a number of unfortunate consequences. Among these number the appearance of unanticipated necrosis of the mandible and/or maxilla and similarly unanticipated (or almost unanticipated) spontaneous fractures of the subtrochanteric femur. There followed a vigorous response by the medical community to these cataclysmic events. This response was exemplified by the convening, after a number of years of case-reports multiplying in the literature, by the American Society of Bone and Mineral Research, of select groups of concerned physicians and scientists tasked with evaluating the origins of these problems.
\end{abstract}

At no time did these so-called Task Forces acknowledge clearly and unambiguously that the preponderance of evidence was consistent with the cause of these dramatic effects being the associated use of the bisphosphonate drugs. The purpose of this opinion paper is to show that the problem of assigning causation, a conundrum which has resulted in contrary opinions of many eminent philosophers, has indeed been convincingly elucidated in a manner that has received acceptance and recognition in the field of applied medicine, and furthermore, to show the application of this technique to the question of the causation of the effects "associated" with the bisphosphonates, with particular reference to the femur fractures mentioned above.

\section{Introduction}

Osteoporotic bone fragility is the result of a natural process resulting from the loss of estrogen associated with aging. "Osteoporosis" and "osteopenia," as these terms are universally used today, do not conform to the typical definition of disease. Over the last 25 years, the definition of osteoporosis has slowly evolved into a conflicting group of several independent meanings. The most common definition of osteoporosis used today conflates mutually exclusive terms. For many years, the diagnosis of osteoporosis depended upon the prevalence of a fracture. This event, examined in the context of the individual's physical examination, could be interpreted in appropriate circumstances as due to an intrinsic lack of resistance to fracture. i.e., osteoporosis. However, in 1992, a group convened by the World Health Organization (WHO) and meeting in
Milan, agreed to define osteoporosis, not only by a prevalent fracture, but also as a bone density 2.5 standard deviations below that of an average 30-year-old white woman. This decision transformed the treatment of a specific clinical illness into a "disease" based on the supposed discovery of a risk, introducing bone mineral density as a surrogate for clinical disease. Subsequently, "osteopenia" became defined, by default, as a bone density lower than one standard deviation below but greater than 2.5 standard deviations below that of an average 30-year-old woman. The adventitious nature of this introduction of so-called osteopenia into clinical medicine has been publicly acknowledged [1] by one of the most prominent scientists involved in many of the Phase 3 bisphosphonate clinical trials as inappropriate. The decision to use these definition limits 
was arbitrary and was perhaps initially only intended to establish degree of risk, and not to afford those terms diagnostic significance $[2,3]$. Yet these criteria have been widely popularized to imply that millions of apparently healthy persons now have a disease called osteoporosis requiring treatment. These millions were immeasurably increased by the additional attribution of disease status to the mineral densities which were higher than, but close enough to, those assigned to osteoporosis.

\section{The Search for a Cure}

The definition of individuals with an appropriate low-impact prevalent fracture or a newly discovered risk was concurrent with the avid search in the pharmaceutical industry for a treatment. The number of individuals meeting the new definition represented a vastly increased market. Ironically, since that time there has been little, quite sporadic cooperation between specialists concerned with the former definition of osteoporosis (a prevalent fracture resulting from negligible trauma), to wit, the orthopedic surgeons, and the several industries proffering medical treatment. Most of the embrace of these risk-aversive preventatives has been with the specialists who have become ever more familiar with the current emphasis on prevention.

\section{Establishing a Meaningful Definition of Causation}

Sir Austin Bradford Hill was a Professor of Biostatistics at the University of London. In 1965, he gave a plenary lecture [4] at the first meeting of the newly created section of Occupational Medicine of the Royal Society of Medicine. Leaving aside many of the niceties which have complicated this issue over the centuries, he proposed a practical method to enable moving from a recognition of association between factors and disease to the conclusion of causation. This is exactly the step which the Task Forces, convened by the American Society of Bone and Mineral Research (ASBMR) to evaluate the association between the bisphosphonate drugs and the complications of osteonecrosis of the jaw [5] and atypical femur fractures [6,7] declined to take, despite the fact that they finally acknowledged that the evidence for the association was "robust" [7]. Bradford Hill proposed a set of well-defined criteria upon which such a conclusion could be reliably reached. He argued his criteria as sufficient despite the semantics indulged in by a long list of philosophers addressing causation. His point was that once an association has been recognized, and possibly before the frequency of that association has been established and almost certainly before the mechanism of action of the association is known, it was still possible to posit as causative relationship if certain criteria could be satisfied. The American Food and Drug Administration has recognized satisfaction of the Bradford Hill [8-10] criteria as a litmus test for drug side-effect causation. The author has observed that the use of the Bradford Hill analysis has increased markedly at the FDA in the interval since the ASBMR Task Force reports. The examples noted are just a random sample of this use.

\section{Application of the Bradford Hill Criteria}

The criteria proposed by Bradford Hill are as follows:

a) Strength of Association

b) Consistency of the Association

c) Specificity

d) Temporality

e) Biological Gradient

f) Plausibility

g) Coherence

h) Experiment

i) Analogy

The Association requirement is met because bisphosphonate use is associated with a detectable increase in the incidence of nonhip femoral fractures. As noted above, the second ASBMR Task Force has recognized this association and called it "robust" [7]. These sorts of datasets have continued to accumulate. As an illustration: One meta-analysis [11] of all the association (incidence) studies which could be found in the literature from 1990-2012 using the ASBMR criteria with all other subtrochanteric/femoral shaft (ST/FS) fractures as well found a relative risk of these fractures in bisphosphonate-exposed individuals of 1.70 times that of unexposed individuals. If the femoral fractures were confined to the more restrictive ASBMR (2009) definition, that relative risk increased to 11.78

Evaluation of all ST/FS fractures without reference to the ASBMR criteria (which were not yet published) in the entire Spanish medical database from 2005-8 inclusive [12] disclosed an incidence of such fractures in ever-users vs never-users of bisphosphonates of 4.3 -fold. In users of 3 or more years, the incidence increased to 9.6-fold

There was 46 times greater bisphosphonate use in ST/FS fracture patients in two major Swedish hospitals over 1 year than in comparable patients who were not using the drugs [13]. This ratio was later verified for the entire nation [14]. The Consistency requirement looks to independent observers to be found to report similar experiences with the side-effect in question. The finding of the association has been consistent in many independent studies and separate venues. The ASBMR Task Force [6,7] summarized independent reports from 17 different countries. The wide difference of incidence reported from various sources has been attributed to differences in data collecting techniques with actual radiographic examination [15-17] generally finding a greater incidence than retrospective review of diagnostic codes. Some of these differences may also be attributable to observer bias, as the 
challenge of these fractures has occasioned intensely defensive reactions in some quarters.

The Specificity requirement is met, not because bisphosphonates are the only cause of these femoral fractures, but because most of the causes of these fractures share the same end point to their common mechanism of action. Bradford Hill [4], the FDA-acknowledged authority in this matter, specifically warns that the mechanisms producing any given side effect may be similar even if quite disparate agents or conditions are causative, thus multiple causes may deceptively appear to challenge specificity. In fact, in my opinion, this particular specificity of mechanism is a perfect demonstration of what Bradford Hill stated would illustrate satisfaction of the requirement. A brief discussion of some proposed mechanisms of action will be found below, but note is taken here that atypical femur fractures have also been reported in association with non-bisphosphonate medications inducing the same or greater degree of antiresorptive remodeling suppression as the bisphosphonates. Denosumab, one of these medications, is also associated with jaw osteonecrosis and atypical femoral fractures similar to the bisphosphonates [18].

Temporality is met because bisphosphonate-associated fractures occur after the ingestion or administration of bisphosphonates rather than in persons never using the drugs. One analysis [19] showed a steadily increasing incidence of very specific femur fractures since 1999 despite the lack of apparent increase in the hip fracture incidence. A U.S. National Institutes of Health analysis showed that non-hip fractures are increasing while hip fractures are decreasing, even in the face of a $50 \%$ decrease in the use of bisphosphonates over the study interval [20].

Satisfaction of the Biological Gradient depends on an adequate understanding of the metabolism of the bisphosphonates. The bisphosphonate medication used for osteoporosis are not metabolized and a long residence in the skeleton is maintained. The terminal half-life has been shown to be around 10 years. This expression refers to the persistence of the drug after re-dosing is stopped [21]. This almost unique situation means that gradient issues must be understood through duration of therapy, not dose. The pharmacokinetics of the bisphosphonates differ qualitatively from the typical kinetics of drug metabolism.

Any dose saturates available binding sites, but only additional doses expose new sites to saturation, the prior dose remaining where it attached. The burden of the drug thus accumulates over time [22]. However, the reported decreasing risk of a second attributable complete fracture if the drug is stopped when the fracture occurs is not necessarily evidence of a withdrawal gradient $[23,24]$. This risk decrease is clearly not connected with a meaningful reduction of the drug burden, but rather with the circumstances surrounding the development of the second fracture. Most complete ST/FS fractures initiate laterally as insufficiency incomplete cracks [25]; the potent stimulation of resorption and formation activity which normally (in the absence of bisphosphonate) combats progress of such a fracture line is inhibited by circulating bisphosphonate which is only present to a large degree when re-dosing is occurring. The occurrence of the initial fracture is likely associated most times with cessation of re-dosing. If it is not, the incidence of a second fracture is greater [26].

A very well-done recent study [27] establishes beyond a reasonable double the relationship between duration of therapy and risk. The Plausibility requirement is met by the many consistent mechanisms whereby the use of the drug could result in bone fragility. The ASBMR Task Force has acknowledged seven plausible mechanisms of action [6,7]. All of these are quite reasonable possibilities. One of the plausible mechanisms alluded to but not expanded in the ASBMR Task Force report relates to an important anatomic parameter long recognized to influence the toughness or energy-to-fracture of bone $[28,29]$. The bone mineral density distribution is a measure of the heterogeneity of the bone mineral matrix [30]. The wider the distribution, the more varied the hemi osteon and osteon density will be. Bone remodeling is a discrete and localized process which is initiated at new locales continuously, each completed remodeling being followed by a continuous but not linear mineralization influx into the tiny newly minted bone packets over a period of time often measured in years. As the initiation process unrolls, these micro loci will necessarily have differing mineral densities depending on how much time has passed since mineralization began.

The result is a three-dimensional patchwork of tiny bone parcels which differ in density from their immediate neighbors. These interfaces between these new parcels, quite appropriately termed "cement lines", act as barriers to crack propagation [31]. Furthermore, the lateral femoral shaft in untreated individuals in the steady state harbors more intrinsic microdamage than the medial shaft [32-34]. This sets up the lateral shaft for a fracture and probably results from and adds to the anatomic stress concentration long known to maximize there [35]. Bisphosphonatetreated patients have a more homogeneous bone due to the marked decrease in new remodeling initiations and the resultant permitted movement of all parcel densities toward a uniform maximum. In addition to this, the femoral shaft has been shown [35] to be more homogeneous than the hip to begin with, and exaggeration of this tendency by bisphosphonates has been shown by biopsy of fractures in that region during orthopedic repair. All of these factors combine to make it very plausible indeed that the ST/FS should be the expected major location of these breaks.

That said, however, it is important to note that there is no consensus that the bisphosphonate effects eventuating in insufficient bone are confined to the femur. Indeed, the original poster [36] presentation revealing this problem to the world in 2003 described similar events in other cortical locations. Around $30 \%$ of the cases I reviewed with Jennifer Schneider in 2012 [26] also sustained 
fractures of the metatarsal bones and we also had occasion later to review many other non-femur sites where the same general fracture types have occurred under the influence of the bisphosphonates [37]. In addition to these and the ASBMR-cited mechanisms, several others may be cited. A plausible theory has been advanced [38] that micro-crack propagation through the homogeneous medium created by chronic bisphosphonate therapy can result in one master crack that may propagate unrestrained to eventuate into a macro- fracture. The lateral initiation particular site of the injury is the result of the maximum standing stress produced in this area due to the shape of the femur. This distribution of force has been well-understood by anatomists for the last hundred years [35].

Many workers in this field, especially in the Scandinavian countries, suggest that the origin of all these fractures is in a form of stress (un-displaced) fracture that differs from the athletic stress fracture in that the latter occurs in adequate bone subjected to chronic excessive force whereas the former occurs in bone rendered inadequate as mentioned above and subjected only to normal force. [39] For this reason, the term "insufficiency fracture" has become favored as it better describes the operative mechanism. The coherence requirement is met because there is no conflict between these mechanisms and the laws of physics and biology. The reported mechanisms have been the product of application of the scientific method in reputable laboratories around the world.

Experiments performed at the material level indicate a decreased toughness of the bone after bisphosphonate exposure [40]. Femur fractures have been demonstrated in bisphosphonatetreated dogs [41]. Bisphosphonates have been experimentally shown to increase the proportion of non-enzymatic glycation products (crosslinks) in bone collagen, an effect associated with decreasing toughness [42]. Bisphosphonates have been shown to decrease cortical modeling, an effect which likely compromises the integrity of the femoral shaft [43]. Animal experiments have also shown [44] that the decrease in bone toughness occasioned by a bisphosphonate is in experimental animals can be reversed by prior or concomitant treatment with corticosteroids, thus quite possibly exonerating corticosteroids from contributing to the fracture problem even though the impact of steroids on the formation phase of bone is well known. Animal experiments have also shown that the presumed inverse correlation between bone mineral density and fracture risk is misleading and false [45]. Finally, physical chemical experiments have been [46] and can be conducted that may confirm that the exposure of bone to phosphorus smoke results in the binding of pyrophosphoric acid, a bisphosphonate analog, to human bone, thus reproducing the femur fragility occurring in one of the analogous contexts mentioned below.

The Analogy requirement was left somewhat optional by Bradford Hill as he recognized that analogies might not always be available. The example he gave was rather weak. But there have been some striking analogies to the drug-induced osteonecrosis and atypical femur fractures pointed out once an understanding developed that effects of bisphosphonates paralleled those of the analogous pyrophosphoric acid [47]. The discovery of the antiresorptive effects of the drugs was preceded by discovery of the antiresorptive effects of pyrophosphoric acid in physiologic conditions in three laboratories in the interval 1968-1980 [48-50]. The Swiss research group of Herbert Fleisch essentially abandoned their interest to this specific point, both reluctantly [51] and recently, more decidedly [52]. These scientists moved on to develop and define the bisphosphonate analogs - work that still continues today under the direction of the successors to Fleisch. Osteopetrosis results from several genetic errors all effecting a deficit in bone resorption. Since the bisphosphonates used in osteoporosis operate chiefly as antiresorptive depressing bone resorption, if this effect is exaggerated enough, an osteopetrotic state might develop. Such a case has been described [53], in the first year that attention was called to the most serious of the drug side-effects and followed closely [54] over many years. Furthermore, atypical femur fractures resembling those caused by the bisphosphonates, have been shown to occur [55] in genetic osteopetrosis in the absence of bisphosphonate treatment. Ironically, treatment of experimental animals with bisphosphonates had been shown [56] many years earlier to induce an osteoporotic state resembling a mouse genetic strain know as grey lethal osteopetrosis.

Pyrophosphoric acid is an abundant product of human metabolism. As described above, it was research in the chemistry and physiology of pyrophosphoric acid that led to the interest in bisphosphonates, which are structurally analogous to it. Pyrophosphoric acid and the bisphosphonates share the pharmacologic properties of antiresorption and antimineralization. Like the osteopetrosis described above, there is a group of human genetic anomalies which result in increased levels of pyrophosphoric acid due to an inherited enzyme dysfunction which normally metabolizes the moiety. This syndrome, in the least extreme form - adult hypophosphatasia, has been recognized [57] as producing fractures that resemble the atypical femoral fractures caused by the bisphosphonates. Again, this presents an excellent satisfaction of Bradford Hill's requirement of rational analogy. Again ironically, the Fleisch group studied [58] this syndrome secondary to their original interest is pyrophosphoric acid. They quantified the increased levels of pyrophosphoric acid in affected individuals, but they never speculated, at least in print, as to the possibility of induction of the fractures suffered in hypophosphatasia by treatment with their acknowledged analog.

One more analogy is currently available. Workers, exposed to the fumes of burning white phosphorus, in phosphorus match factories in the latter half of the 19th century occasionally developed osteonecrosis of the jaw and/or low trauma femoral fractures. Analogous to the hypophosphatasia victims, who were exposed internally to excess pyrophosphoric acid, these workers were exposed to an external source of the same molecule, since the fresh smoke from burning white phosphorus has been shown 
[59] to consist largely of the two substances shown by Fleisch [48] to have antiresorptive properties: pyrophosphoric acid and mixed polyphosphates. The details of this interesting analogy have been presented [60] elsewhere.

\section{Conclusion}

An objective evaluation of the facts found in the published literature has been applied to the question of causation by bisphosphonates of the well-documented problems of jaw osteonecrosis and atypical femur fractures associated with the drugs. This task was undertaken because of the remarkable reluctance of the generally recognized if heterogeneous authority of the American Society of Bone and Mineral Research to undertake this important step. There seems to be no doubt that the Bradford Hill criteria for the confirmation of causality can be comfortably satisfied despite insufficient efforts appearing from time to time in the medical and scientific literature. The author believes that a working knowledge of phosphorus chemistry is a key factor allowing understanding of the answers provided by the extant facts with respect to the fundamental question. Philosophical semantic quibbles aside, the accepted methodology proposed by Sir Austin Bradford Hill more than half a century ago and endorsed and utilized by the American Food and Drug Administration produces, when properly applied, proof that the bisphosphonate drugs are the cause of the osteonecrosis and atypical femur fractures associated with their use.

\section{References}

1. Cummings S (2012) Osteopenia: How we created a "disease," and how to manage patients with osteopenia. JDSA 17(1): 15.

2. Kanis J A, Oden A, Johansson H, Borgström F, Ström O, et al. (1994) FRAX and its applications to clinical practice. Bone 44: 734-743.

3. (1994) Assessment of Fracture Risk and its Application to Screening for Postmenopausal Osteoporosis: Synopsis of a WHO Report Osteoporosis Int 4: 368-381.

4. Bradford Hill A (1965) The Environment and Disease: Association or Causation? Proceedings of the Royal Society of Medicine 58: 295-300.

5. Khosla S, Burr D, Cauley J, Dempster DW, Ebeling PR, et al. (2007) Bisphosphonate-Associated Osteonecrosis of the Jaw. Report of a Task Force of the American Society of Bone and Mineral Research. J Bone Miner Res 22: 1479-1491.

6. Shane E, Burr D, Ebeling PR, Abrahamsen B, Adler RA, et al. (2010) Atypical subtrochanteric and diaphyseal femur fractures: report of a task force of the ASBMR. J Bone Miner Res 25: 2267-2294.

7. Shane E, Burr D, Abrahamsen B, Adler RA, Brown TD, et al. (2013) Atypical subtrochanteric and diaphyseal femoral fractures: Second report of a task force of the ASBMR. J Bone Miner Res 29: 1-23.

8. (2019) Meeting of the Circulatory System Devices Panel Regarding Paclitaxel-Coated Products Indicated for Peripheral Arterial Disease (PAD) June 19-20: 2019.

9. (2016) Arsenic in Rice and Rice Products Risk Assessment Report Center for Food Safety and Applied Nutrition Food and Drug Administration US Department of Health and Human Services March 2016.

10. (2017) Causal Inference and Control for Confounding. Office of Surveillance and Epidemiology Center for Drug Evaluation and Research US Food and Drug Administration July 10, 2017.
11. Gedmintas L, Solomon DH, Kim SC (2013) Bisphosphonates and Risk of Subtrochanteric, Femoral Shaft, and Atypical Femur Fracture: A Systematic Review and Meta-Analysis. J Bone Miner Res 28(8): 17291737.

12. Erviti J, Álvaro Alonso, Belén Oliva, Javier Gorricho, Antonio López (2013) Oral bisphosphonates are associated with increased risk of subtrochanteric and diaphyseal fractures in elderly women: a nested case-control study. BMJ Open 3(1): e002091.

13. Schilcher J, Michaëlsson K, Aspenberg P (2011) Bisphosphonate Use and Atypical Fractures of the Femoral Shaft. N Eng J Med 364(18): 17281737.

14. Schilcher J, Veronika Koeppen, Per Aspenberg, Karl Michaëlsson (2014) Risk of Atypical Femoral Fracture During and After Bisphosphonate Use. N Eng J Med 371(10): 974-976.

15. Dell RM (2010) A Retrospective Analysis of all Atypical Femur Fractures Seen in a Large California HMO from the Years 2007 to 2009. Oral Presentations, Presentation and Abstract Number: 1201 ASBMR Annual Meeting October p. 15-19.

16. Tripto-Shkolnik L, Segal E, Jaffe A, Ish Shalom S, Bachrach R (2015) Atypical Femoral Fractures: Radiological Evaluation and Bisphosphonate Exposure. IMAJ 17(9): 554-558.

17. Lian K, Trollip J, Sandhu S, Moosavi M, Gill A (2016) Audit of Atypical Femoral Fractures and a Description of Some of Their Features. Canadian Association of Radiologists Journal 67(1): 69-75.

18.(2010) See, for example, the FDA-mandated Risk Evaluation and Mitigation Strategy for Prolia $\AA$ (denosumab) approved 06/10/2010 with subsequent modifications.

19. Feldstein AC, Black D, Perrin N, Rosales AG, Friess D (2012) Incidence and Demography of Femur Fractures with and Without Atypical Features. J Bone Miner Res 27(5): 977-986.

20. Jha S, Wang Z, Laucis N, Bhattacharyya T (2015) Trends in Media Reports, Oral Bisphosphonate Prescriptions and Hip Fractures 19962012: An Ecological Analysis. J Bone Miner Res 30(12): 2179-2187.

21. Kahn SA, Kanis JA, Vasikaran S, Kline WF, Matuszewski BK (1997) Elimination and Biochemical Responses to Alendronate in Postmenopausal Osteoporosis. J Bone Miner Res 12(10): 1700-1707.

22. Shkolnikova J, Flynn J, Choong P (2012) Burden of bisphosphonateassociated femoral fractures. ANZ J Surg 83(3): 175-181.

23. Schilcher J, Veronika Koeppen, Per Aspenberg, and Karl Michaëlsson (2015) Risk of atypical femur fracture during and after bisphosphonate use. Full report of a nationwide study. Acta Orthopaedica 86(1): 100107.

24. Schilcher J, Michaëlsson K, Aspenberg P(2011) Bisphosphonate Use and Atypical Fractures of the Femoral Shaft. N Eng J Med 364(18): 17281737.

25. Aspenberg P, Jörg Schilcher, Anna Fahlgren (2010) Histology of an undisplaced femoral fatigue fracture in association with bisphosphonate treatment frozen bone with remodeling at the crack. Acta Orthopaedica 81(4): 460-462

26. Schneider JP, Hinshaw WB, Su C, Solow P (2012) Atypical femur Fractures: 81 Individual Personal Histories. J Clin Endochrinol Metab 97(12): 4324-4328.

27. Lo JC, Grimsrud CD, Ott SM, Chandra M, Hui RL (2019) Atypical femur fracture in women increases with the duration of treatment. Osteopor Inter 30(12): 2515-2520.

28. Fyhrie DP, Christiansen BA (2015) Bone Material Properties and Skeletal Fragility. Calcif Tissue Int. 97(3): 213-228.

29. Yang K (2012) Correlation between Pamidronate Concentration in Bone and Biomechanical Properties of Rat Femora in Osteoporotic Fracture Model. ASBMR Annual Meeting Abstract P43. 
30. Parfitt AM (1996) Skeletal Heterogeneity and the Purposes of Bone Remodeling Implications for the Understanding of Osteoporosis. In Osteoporosis Academic Press Pp. 315-329.

31. Roschger P, Paschalis EP, Fratzl P, Klaushofer K (2008) Bone mineralization density distribution in health and disease. Bone 42(3): 456-466.

32. Burr DM, Allen MR (2015) Foreword: Calcified Tissue International and Musculoskeletal Research Special Issue: Bone Material Properties and Skeletal Fragility. Calcif Tissue Int 97(3): 199-200.

33. Allen MR, Burr DM (2005) Human femoral neck has less cellular periostium, and more mineralized periostium, than femoral diaphyseal bone. Bone 36(2): 311-316.

34. Donnelly E, Meredith DS, Nguyen JT, Boskey AL (2012) Bone tissue composition varies across anatomic sites in the proximal femur and the iliac crest. J Orthop Res 30(5): 700-706.

35. Koch JC (1917) The Laws of Bone Architecture. Amer J Anatomy. 21(2): 177-298.

36. Odvina CV (2003) Poster SU344 Adynamic Bone Disease during Bisphosphonate Therapy: Should We Be Concerned? Annual meeting of the ASBMR.

37. Hinshaw WB (2017) Bisphosphonate-associated Atypical Fractures that are not "Atypical Femoral Fractures" Brit J Med \& Med Res 19: 1-17.

38. Ettinger B, Burr DB, Ritchie RO (2013) Proposed pathogenesis for atypical femoral fractures: Lessons from material research. Bone 55(2): 495-500.

39. Aspenberg P (2016) Atypical fractures, a biased perspective. Injury Int J. Care Injured 47(S1): S28-S30.

40. Smith ER, Allen MR (2013) Bisphosphonate-induced reductions in rat femoral bone energy absorption and toughness are testing-rate dependent. J Orth Op Res 31(8): 1317-1322.

41. Flora L (1981) Comparative Skeletal Effects of Two Diphosphonates in Dogs in Bone Histomorphometry: Third International Workshop. In: WSS Jee, AM Parfitt (Eds.), Armout-Montagu, Paris, pp. 389-407.

42. Acevedo C, Bale H, Gludovatz B, Wat A, Tang SY (2015) Alendronate treatment alters bone tissues at multiple structural levels in healthy canine cortical bone. Bone 81: 352-363.

43. Allen MR, Kubek DJ, Burr DB (2010) Cancer Treatment Dosing Regimens of Zoledronic Acid Result in Near Complete Suppression of Mandible Intra-Cortical Bone Remodeling in Beagle Dogs. J Bone Miner Res 25(1): 98-105.

44. Luo TD, Allen MR (2013) Short-courses of dexamethasone abolish bisphosphonate-induced reductions in bone toughness. Bone 56(1): 199- 203.

45. Nielsen SP (2000) The Fallacy of BMD: A Critical Review of the Diagnostic Use of Dual X-ray Absorptiometry. Clin Rheumatol 19(3): 174-183.

46. Hinshaw WB (2010) Unpublished. In vitro exposure of human bone tissue to phosphorus smoke. Additional experimental approaches have been proposed by the author and collaborators in a book to be published by NOVA in 2020 entitled "Another Look at Pyrophosphates".

47. Hinshaw WB, Allyn F DeLong (2016) An Evaluative History of Bisphosphonate Drugs: Dual Physiologic Effects of Pyrophosphate as Inspiration for a Novel Pharmaceutical Class. J Osteopor Article ID 1426279.

48. Fleisch H, Straumann F, Schenk R, Bisaz S, Allgöwer M (1966) Effect of condensed phosphates on calcification of chick embryo femurs in tissue culture. Am J Physiol 211(3): 821-825.

49. Orimo H, Fujita T, Yoshikawa M (1969) Pyrophosphate Enhancement of Thyrocalcitonin Effect in Tissue Culture. Endocrinol Japon 16(4): 415421

50. DeLong A, Feinblatt J, Rasmussen H (1971) The Effect of Pyrophosphate Infusion on the Response of the Thyroparathyroidectomised Rat to Parathyroid Hormone and Adenosine-3,5-cyclic Monophosphate. Calcif Tiss Res 8(2): 87-95.

51. Jung A, Bisaz S, Fleisch H (1973) The Binding of Pyrophosphate and Two Diphosphonates by Hydroxyapatite Crystals. Calcif Tiss Res 11(4): 269280.

52. Orriss IR, Arnett TR, Russell RG (2016) Pyrophosphate: A key inhibitor of mineralisation. Curr Opin Pharmacol 28: 57-68.

53. Whyte MA, Wenkert D, Clements KL, McAlister WH, Mumm S (2003) Bisphosphonate-Induced Osteopetrosis. N Engl J Med 349(5): 457-463.

54. Whyte MA, Mc Alister WH, Novack DV, Clements KL, Schoenecker PL (2008) Bisphosphonate-Induced Osteopetrosis: Novel Bone Modeling Defects, Metaphyseal Osteopenia, and Osteosclerosis Fractures After Drug Exposure Ceases. J Bone Miner Res 23(10): 1698-1707.

55. Yates CJ, Bartlett MJ, Ebeling PR (2011) An atypical subtrochanteric femoral fracture from pycnodysostosis: a lesson from nature. J Bone Miner Res 26(6): 1377-1379.

56. Reynolds JJ, Murphy H, Mühlbauer RC, Morgan DB, Fleisch H (1973) Inhibition by Diphosphonates of Bone Resorption in Mice and Comparison with Grey-Lethal Osteopetrosis. Calcif Tiss Res 12(1): 5971.

57. Whyte MP (2009) Atypical Femoral Fractures, Bisphosphonates, and Adult Hypophosphatasia. J Bone Miner Res 24(6): 1132-1134.

58. Russell RGG, S Bisaz, A Donath, DB Morgan, H Fleisch (1971) Inorganic Pyrophosphate in Plasma in Normal Persons and in Patients with Hypophosphatasia, Osteogenesis Imperfecta, and Other Disorders of Bone. J Clin Invest 50(5): 961-969.

59. Spanggord RJ (1985) Environmental Fate of White Phosphorus/Felt and Red Phosphorus/Butyl Rubber Military Screening Smokes. U. S. Army Medical Research and Development Command, Fort Detrick, Frederick, Maryland 21701-5012

60. Hinshaw WB (2015) Recognition of the Causative Agent of "Phossy Jaw" and "Fragile Femur" in Fumes Arising from White Phosphorus. Phosphorus, Sulfur, and Silicon and the Related Elements 190(12): 2082-2093.
ISSN: 2574-1241

DOI: $10.26717 /$ BJSTR.2020.25.004276

William Banks Hinshaw. Biomed J Sci \& Tech Res

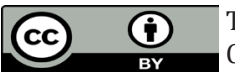

This work is licensed under Creative Commons Attribution 4.0 License

Submission Link: https://biomedres.us/submit-manuscript.php

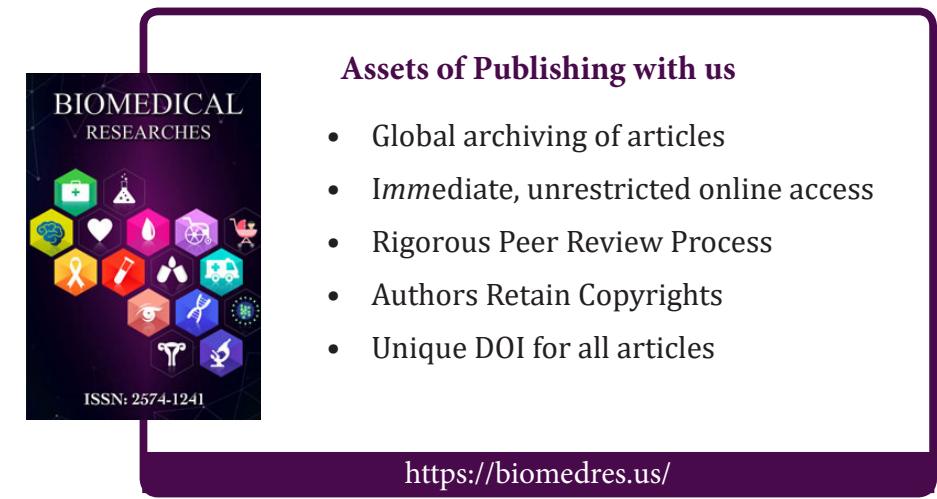

